\title{
Risk-based security-constrained economic dispatch in power systems
}

\author{
Qin WANG, Aimin YANG, Fushuan WEN (ه), \\ Jihong LI
}

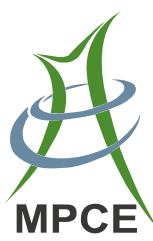

\begin{abstract}
Based on a new perspective in coordinating with the traditional " $N-1$ " criteria and system risk, a real-time electricity market model is presented, in which the system risk is employed to model the system's overall security level. This new model is called the risk-based security-constrained economic dispatch (RB-SCED). Relative to the securityconstrained economic dispatch (SCED) used in the power industry today, the RB-SCED finds more secure and economic operating conditions. It does this by obtaining solutions that achieve a better balance between post-contingency flows on individual branches and the overall system risk. The method exploits the fact that, in a SCED solution, some postcontingency branch flows which exceed their limits impose little risk while other post-contingency branch flows which are within their limits impose significant risk. The RB-SCED softens constraints for the former and hardens constraints for the latter, thus achieving simultaneous improvement in both security and economy. In this work, the basic concept and the mathematical formulation of the RB-SCED model are systematically described. Experimental results on a 9-bus system and the ISO New England actual system have demonstrated the advantages of RB-SCED over SCED.
\end{abstract}

Keywords Economic dispatch, Security-constrained economic dispatch (SCED), Risk-based securityconstrained economic dispatch (RB-SCED), Contingency, Power system

Received: 13 November 2012/Accepted: 1 June 2013/Published online: 29 August 2013

(C) The Author(s) 2013. This article is published with open access at Springerlink.com

Q. WANG, Iowa State University, Ames, IA 50011-2151, USA

A. YANG, South China University of Technology, Guangzhou 510640, China

F. WEN, Zhejiang University, Hangzhou 310027, China

$(\bowtie)$ e-mail: fushuan.wen@gmail.com

J. LI, Zhejiang Electric Power Corporation, Hangzhou 310007, China

\section{Introduction}

Risk assessment (RA) has been widely used in other industries, such as nuclear, aerospace, oil, food, public health, information technology, and financial engineering. It is an emerging new topic in power engineering. Although the successful application of RA in other areas could provide valuable experience for the implementation of risk-based approach in power systems, the definition as well as the meaning of risk is quite different. In fact, the risk assessment has a wide-ranging content. Traditional popular RA methods, such as mean-variance, Value-at-Risk and real operation approaches have been used in business and finance areas - this kind of risk approach primarily takes an angle from the economic perspective. The intent of this paper, however, is to discuss the models, methods and applications of engineering risk in physical power systems. The major difference between engineering risk and financial risk lies in their sources of uncertainties. For example, the financial risk is rooted from uncertainties of credit, investment and market liquidity, etc., while the engineering risk comes from failures of equipment, behaviors of persons and weather conditions, etc. In addition, the engineering risk should be in accordance with the physical law of power systems.

According to an IEEE standard [1] risk can be calculated as the product of the probability of a contingency occurrence multiplied by the consequence of that contingency. In real world, both the probability and the consequence of an event occurrence are difficult to quantify. Thus, the risk management (RM) method, whose purpose is to identify, assess and prioritize risk, should be studied to minimize, monitor and control the probability and/or sequence of the unexpected events. There should be at least three tasks for risk management in power systems:

1) Setting up standards or measures for quantifying the risk. 
2) Determining the acceptable risk levels for power system operation or planning.

3) Finding the effective mechanisms to reduce the risk.

The application of risk management in power systems is motivated by a perceived increase in the frequency at which power system operators are encountering high stress in bulk transmission systems and the corresponding need to improve security monitoring of these networks. Traditional security assessment approach in power systems tries to capture risk with rules like the so-called " $N-1$ " security criteria. Generally, the security-assessment method can be divided into two groups: deterministic and probabilistic approaches. The deterministic approach, i.e., the " $N-1$ " security-based decision method, is a worse-scenario approach that is, any normal state is acceptable, and the other states are not acceptable. The weakness of the approach is that it lacks a quantitative method to measure the security level and distinguish between the states. Consequently, rough rules of thumb are adopted in the decision process. Most importantly, the lack of a security level index disguises the fact that it is hard to distinguish the alert state and the normal state, both of which the equality and inequality constraints are satisfied and unexpected events may cause undesirable consequences. Thus, the risk index is a good metric to distinguish the operating conditions of different states, and quantify the likelihood and/or severity of undesirable consequences.

The deterministic approach has been applied in the industry for a long time [2-5]. In the deterministic framework, the system security analysis is performed in terms of the thermal loading of system elements [6], voltage and frequency variations for both transient and steady states [7]. The basic idea is that the system is able to withstand a set of selected contingencies, which are supposed to have a significant likelihood of occurrence. Although the deterministic approach has well-served the industry on supporting the economic and secure operation of power system in the past decades, there has been a tangible price to pay for applying it: the solution tends to be conservative because it tends to focus on the most severe events. Consequently, it may lead to such situations that existing facilities cannot be fully explored (in operation), or system resources be overbuilt (in planning). Another weakness of the deterministic approach is that there is no index to measure the system security level, thus it is difficult to integrate security into the economic decision-making process.

In the past few years, outage events of power systems happened many times all around the world. According to [8], the national cost of power interruptions in USA is about $\$ 80$ billion annually. Some severe power outages have happened recently. For example, the Northeast Blackout occurred on August 14, 2003 in North America area affected an estimated 10 million people in Canada and 45 million people in eight states of USA. On November 10, 2009, a power outage occurred throughout much of Brazil and entirety of Paraguay (for a short time) affected an estimated 60 million people. These severe outages of power system incentives re-examined the single-contingency criterion (" $N-1$ " principle) that has served the industry for the past decades. The " $N-1$ " criterion cannot be sufficient to guarantee the system at a reasonable security level. On the other hand, it is generally accepted that implementing " $N-2$ " or even higher " $N-k$ " $(k \geq 3)$ security principle may cause excessive financial and computational costs for utility companies. Consequently, one attractive and applicable alternative adopts risk-based approach in the planning and operations of power systems.

The probabilistic approach roots from the nature of probabilistic behaviors in power systems. For example, the random failure of power equipment is usually beyond the control of system personnel; loads will always be uncertain and it is impossible to forecast the load exactly precise. It is known that the probabilistic methods have been used as powerful tools in various kinds of decision-making process [9-17]. In [18-22], the authors focus on developing risk indices, which consider both the likelihood and the severity of events, to capture the probabilistic nature of power systems. One of the most attractive implementation of the proposed methodology is to perform the on-line risk-based security assessment (RBSA) [23-26]. Compared with traditional online security assessment who always performs security assessment on a past condition (i.e., the last state-estimation), the RBSA has the feature that it performs security assessment under a nearfuture condition. One significant advantage of this feature lies in that the decision based information, from the assessment, corresponds to the time frame in which the decision is effective [25]. Although the deterministic methods are still dominated in the industry, there is consensus that using probabilistic approach has a great potential to improve on analysis and decision-making.

This paper introduces a new concept of dispatch method, called the risk-based security-constrained economic dispatch (RB-SCED), and explores its application in power systems. The work is an extension of previous works [27-33]. In the rest of this paper, we introduce the basic concept and mathematical formulation of RB-SCED model, and test the model in two systems to illustrate the benefits of RB-SCED over SCED.

\section{The need of risk-based approach under uncertainty}

Risk is a measure of uncertainties. The main motivation of risk-based approach lies in the fact that the power 
industry is increasingly facing more uncertainties, which have brought challenges to the security of power systems. On July 21, 2011, the Federal Energy Regulatory Commission (FERC) issued Order 1000 which supports many states in USA to focus upon the following three topics of future power system: energy efficiency, demand response and smart grid. All of them will increase the uncertainties of the systems. Energy efficiency means using less energy to provide the same service. Some activities suggested by the International Energy Agency (IEA) to save energy, such as "turning off the lights when not using it" and "using a power strip to turn off stand-by power in electronics and appliances [34]," will increase the uncertainty on human usage of electricity. Demand response is a mechanism to encourage customers reducing their electricity consumption in response to market prices. The Independent System Operators (ISOs) may even execute load shedding according to the demand side bidding price when necessary. The uncertainties, from both customer behaviors and demand side load forecasting, make the operation of power system more complicated. The smart grid has a broad range of contents. Although its definition is various, the following characteristics are generally accepted to form the future smart grid environment: higher penetration of renewable generation resources, deployment of advanced electricity storage and peak-shaving technologies including plug-in hybrid electric vehicles (PHEVs), provision to consumers with timely information and control options, increased use of information technology to improve reliability, security and efficiency of the electric power grid. Obviously, the smart grid environment will bring new challenges to the operation of power systems. All these factors will force power utilities to operate and plan the system closer to the limits, thus leading to more stressed operation conditions.

The risk-based approach is an emerging new direction that is studied and beginning to be used in power system planning [35, 36] and maintenance [37, 38]. Most of previous work focus on the Risk-based Security Assessment (RBSA). Researches on the application of risk-based approach for real-time operation are rare. The major reasons are that the "N-1" principle applied in RBSA is simple to implement and to understand, and our operating paradigm and tools have not evolved to enable observation of its weakness. References [39, 40] proposed the frameworks of risk-based approach application for power grids, but did not provide details on how to realize it. A risk-limiting dispatch under smart-grid environment was proposed in [41]. Although it has provided models taking into account the stochastic nature of renewable sources and the demand response, it may have computational issues to extend the model in real-world large-scale power systems. Our work on RB-SCED is an early attempt to prompt the application of risk-based approach on power systems.

\section{Basic concept of risk-based economic dispatch}

In this section we will present the basic concept of RBED, without exploring the details of formulations and computational strategies. Firstly, we introduce the socalled security diagram proposed in [28], as shown in Figs. 1 (a) and (b). The information provided in these figures is described as follows:

Security regions There are three regions: emergency region, highly-stressed region and less-stressed region, corresponding to the areas with red, yellow and white colors, respectively. If only overload violations are considered, the less-stressed region refers to the circuit loading less than $90 \%$ of the emergency rating; the highly-stressed region corresponds to loadings less than $90 \%$ of the emergency rating; the emergency region corresponds to loadings in excess of emergency rating.

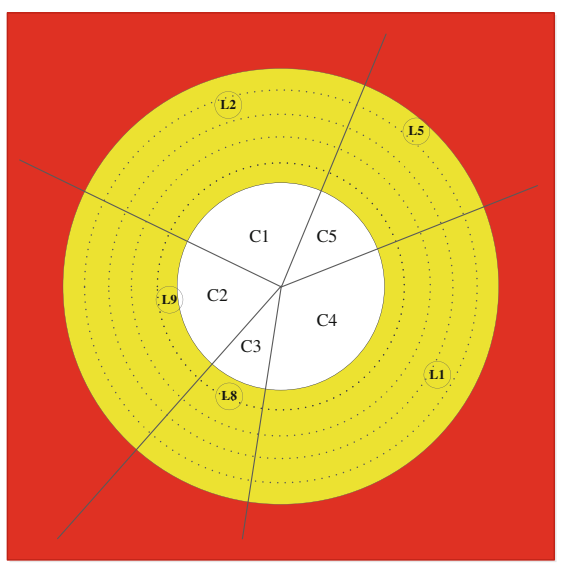

(a) Security diagram for the solution to SCOPF

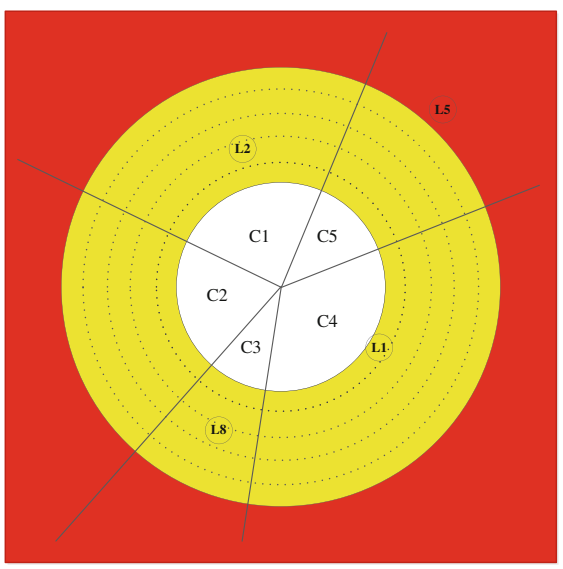

(b) Security diagram for the solution to RBOPF

Fig. 1 Security diagram 
Probability sectors Supposing that for this particular system, there are five post-contingencies: $\mathrm{C} 1$ to $\mathrm{C} 5$. Note that the contingency set in an actual power system is very large, and we may just list the contingencies that bring the system to emergency or highly-stressed regions. The angular spread of each sector is proportional to the contingency probabilities. For example, $\mathrm{C} 4$ has the largest probability among the five.

Severity circles The small circles L1, L2, L5, L8 and L9 represent circuits with post-contingency overload violation or near-violation. In this system, some circuit numbers, such as L3, L4, L6 and L7 are not listed in the figure because these circuits will not cause highly-loaded conditions. The radial distance from the center of the diagram to each small circle is proportional to the extent (severity) of the violation. For example, L1 in Fig. 1 (a) means that flow at circuit L1 is $97.5 \%$ of its emergency rating under contingency $\mathrm{C} 4$.

Figures 1(a) and (b) demonstrate the benefits of RBOPF over SCOPF. In Fig. 1(a), all the post-contingency branch flows will not exceed their contingency ratings, it shows the requirement of the " $N-1$ " principle. However, this is a highly stressed system since some high-probability circles are located close to the red zone. In contrast, the RBOPF in Fig. 1(b) shows that the high-probability circles L1, L2 and L9 move closer to the white zone, at the cost of moving low-probability circles L5 and L8 closer to the red zone. By this way, lower risk is achieved by decreasing severity on high probability violations L1, L2 and L9 while increasing severity of low probability violations L5 and L8. Although one violation L5 exceeds its deterministic limit, the overall system risk is lower.

\section{RB-SCED model}

\subsection{Description of risk index}

Risk is a probabilistic index defined to reflect the severity of the system operation condition [27]. Commonly used indices include overload, cascading overload, low voltage, and voltage instability [30]. In the RB-SCED model, only the risk of overload is considered in accordance with the current real-time dispatch software in electricity market, where DC model is used for base case. Define $E_{0}$ as the system loading condition in normal state and $E_{i}(i=1,2, \ldots, N)$ in contingency states. The risk of the system at certain time $t$ is

$\operatorname{Risk}(t)=\sum_{i=1}^{N} \operatorname{Pr}\left(E_{i}, t\right) \operatorname{Sev}\left(E_{i}, t\right)$

where $\operatorname{Pr}\left(E_{i}, t\right)$ is the probability of state $i$ at time $t$, and $\operatorname{Sev}\left(E_{i}, t\right)$ is its severity at time $t$. A typical expression of the severity function of overload for a single circuit is:

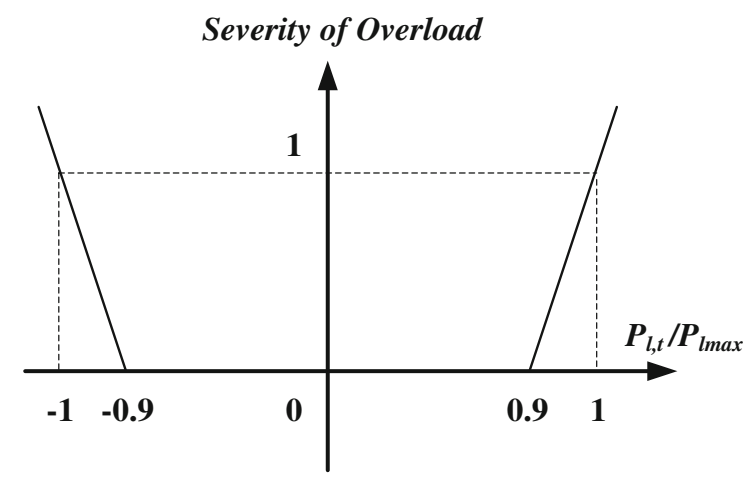

Fig. 2 Severity function of circuit overload

Sev $=\left\{\begin{array}{lc}10 \times\left(-P_{l, t} / P_{l \max }-0.9\right), & -P_{l \max }<P_{l, t}<-0.9 P_{l \max } \\ 0, & -0.9 P_{l \max }<P_{l, t}<0.9 P_{l \max } \\ 10 \times\left(P_{l, t} / P_{l \max }-0.9\right), & 0.9 P_{l \max }<P_{l, t}<P_{l \max }\end{array}\right.$

where $P_{l, t}$ is the power flow of circuit $l$ at time $t$, and $P_{l \max }$ is its transmission limit. The severity function is shown in Fig. 2.

The probability of contingency is computed using statistical measures. To obtain its probability, we need to know the frequency of occurrence of the contingency in a certain time interval. In this paper, we use the methods proposed in [42] and [43] to obtain the probabilities of contingency.

\subsection{Formulations of SCED, RBED and RB-SCED}

The compact form of SCED is formulated as follows:

$$
\begin{cases}\min _{\boldsymbol{x}_{0}, \boldsymbol{x}_{1}, \ldots, \boldsymbol{x}_{N C}, \boldsymbol{u}_{0}} f_{0}\left(\boldsymbol{x}_{0}, \boldsymbol{u}_{0}\right) \\ \text { s.t. } & \boldsymbol{g}_{k}\left(\boldsymbol{x}_{k}, \boldsymbol{u}_{0}\right)=\mathbf{0}, \quad k=0,1, \ldots, N C \\ & \boldsymbol{h}_{k}\left(\boldsymbol{x}_{k}, \boldsymbol{u}_{0}\right) \leq \boldsymbol{h}_{k}^{\max }, k=0,1, \ldots, N C\end{cases}
$$

where $f_{0}$ is the objective function, $k$ is the state of the system while $k=0$ corresponding the normal state and $k=1,2, \ldots$, $c$ corresponding to the post-contingency states, $\boldsymbol{g}_{k}$ and $\boldsymbol{h}_{k}$ are the equality and inequality constraints, respectively, $\boldsymbol{x}_{k}$ is the state vector for the $k$-th configuration, $\boldsymbol{u}_{0}$ is the vector of the control variables in the normal state, and $\boldsymbol{h}_{k}^{\max }$ is the vector of limit for inequality constraints at state $k$.

The benchmark RBED problem can be formulated as follows:

$$
\begin{cases}\min _{\boldsymbol{x}_{0}, \boldsymbol{x}_{1}, \ldots, \boldsymbol{x}_{N C}, \boldsymbol{u}_{0}} f_{0}\left(\boldsymbol{x}_{0}, \boldsymbol{u}_{0}\right) \\ \text { s.t. } & \boldsymbol{g}_{k}\left(\boldsymbol{x}_{k}, \boldsymbol{u}_{0}\right)=\mathbf{0}, \quad k=0,1, \ldots, N C \\ & \boldsymbol{h}_{0}\left(\boldsymbol{x}_{0}, \boldsymbol{u}_{0}\right) \leq \boldsymbol{h}_{0}^{\max } \\ & \sum_{k=1}^{N C} \operatorname{Risk}_{k}\left(\operatorname{Pr}_{k}, \boldsymbol{x}_{k}\right) \leq \text { Risk }_{\max }\end{cases}
$$


where $\operatorname{Pr}_{k}$ is the probability of occurrence for the $k$-th state, $\operatorname{Risk}_{k}\left(\operatorname{Pr}_{k}, \boldsymbol{x}_{k}\right)$ is the corresponding risk computed by given probability $\operatorname{Pr}_{k}$ and state $\boldsymbol{x}_{k}$, Risk $_{\max }$ is the limit of the system security level. Note that the flow constraints for individual circuits at post-contingency are not considered in (4). Instead, it restrains overall security level of the system.

The RB-SCED problem combines the above two models together by introducing two coordination factors $K_{C}$ and $K_{R}$, as indicated in formulation (5):

$$
\begin{cases}\min _{\boldsymbol{x}_{0}, \boldsymbol{x}_{1}, \ldots, \boldsymbol{x}_{N C}, \boldsymbol{u}_{0}} f_{0}\left(\boldsymbol{x}_{0}, \boldsymbol{u}_{0}\right) \\ \text { s.t. } & \boldsymbol{g}_{k}\left(\boldsymbol{x}_{k}, \boldsymbol{u}_{0}\right)=\mathbf{0}, \quad k=0,1, \ldots, N C \\ & \boldsymbol{h}_{0}\left(\boldsymbol{x}_{0}, \boldsymbol{u}_{0}\right) \leq \boldsymbol{h}_{0}^{\max } \\ & \boldsymbol{h}_{k}\left(\boldsymbol{x}_{k}, \boldsymbol{u}_{0}\right) \leq K_{C} \times \boldsymbol{h}_{k}^{\max }, \quad k=1,2, \ldots, N C \\ & \sum_{k=1}^{N C} \operatorname{Risk}_{k}\left(\operatorname{Pr}_{k}, \boldsymbol{x}_{k}\right) \leq K_{R} \times \text { Risk }_{\max }\end{cases}
$$

The choice of $K_{C}$ and $K_{R}$ in (5) enables us to impose control over a tradeoff between security and economics: the higher the $K_{C}$ and $K_{R}$ are, the more economic and less secure the system is; and also a tradeoff between system risk and individual circuit overload: increasing $K_{C}$ may decrease the security of individual circuit while decreasing $K_{R}$ may increase the security of the system, and vice versa. Three different operational models are proposed for RB-SCED: highly secure model (HSM), economic-secure model (ESM), and extra economicsecure model (HEM), where $K_{C}$ is chosen as $1,1.05$ and 1.25 , respectively.

By choosing proper $K_{R}$ and $K_{C}$ according to real-time operation condition, the system can gain significantly economic benefits as well as improve the long-term security level. For example, one may choose the "high security" operation point, $\left(K_{R}, K_{C}\right)=(0.6,1)$, under conditions when high system security is required, such as heavy load and severe weather. Similarly, one may choose a "high economy" operation point, say $\left(K_{R}, K_{C}\right)=(0.5,1.2)$, if the system stress is low. Although a certain degree of postcontingency overloading is allowed, the using "high economy" mode is attractive because of the following three facts:

1) The probability of a contingency occurrence is very low, e.g., the trip of a transmission line may happen only several times during a year, but we have to prevent it in SCOPF model for every hour.

2) Corrective actions can be taken to bring the system back to the normal state, if a contingency occurs, thus guaranting the system security.

3) The degree of post-contingency overloading can be controlled by choosing appropriate $K_{C}$.

\section{Numerical results}

Two representative numerical examples are presented by the proposed approaches: the IEEE 9-bus test system and the ISO New England real system.

\subsection{The IEEE 9-bus test system}

The diagram of the IEEE 9-bus test system is shown in Fig. 3. All the line impedances are indicated in the diagram with per unit values.

The loads at bus 5, 6, and 8 are $125 \mathrm{MW}, 90 \mathrm{MW}$ and $100 \mathrm{MW}$, respectively. For simplicity, we assume the generators only provide one-segment bidding prices, which are 20,40 and $80 \$ / \mathrm{MWh}$ for generator 1,2 and 3 , respectively. The maximum economic outputs of these generators are 150, 200 and $150 \mathrm{MW}$, respectively. The parameters of the transmission lines and transformers, including the impedances, resistances and MW limits are shown in Table 1. Two " $N-1$ " contingencies are considered, i.e., the outages of circuits 4-5 and 6-9. Assuming that their outage probabilities are both 0.01 at certain time $t$, thus the probability of normal state is 0.98 .

It is assumed that this is a lossless network while the loss is constant during the computation. The loss offset is -4.46 MW calculated against reference bus 1 .

Firstly, we solve the SCED model of the system. The cost is $\$ 11,377.8$, and the risk value is 0.9 , which is set as the maximum risk $R_{\max }$. Then, the three different models of RB-SCED, i.e., the HSM model $\left(K_{C}=1, K_{R}=0.5\right)$, the ESM model $\left(K_{C}=1.05, K_{R}=0.75\right)$, and the HEM model $\left(K_{C}=1.20, K_{R}=0.5\right)$, are solved. The results are shown in Tables 2 and 3. Table 4 lists the circuits with flow over $90 \%$ of their limits at both normal and postcontingencies for SCED, RBED and various models of RB-SCED.

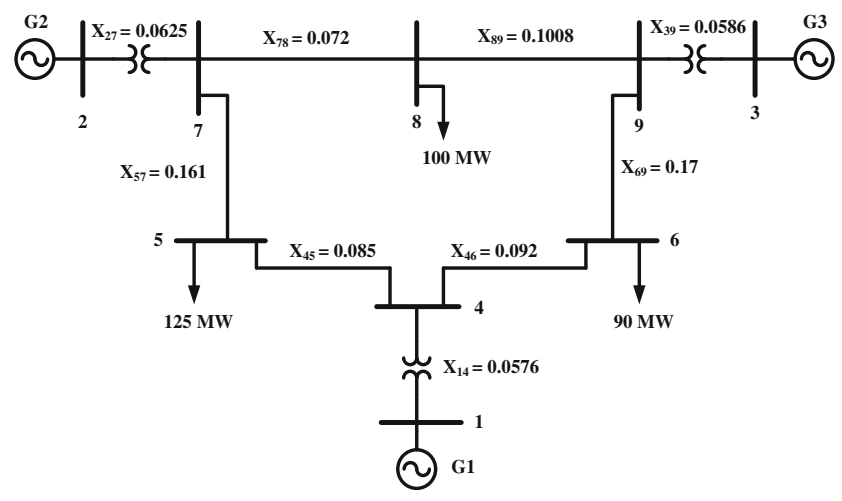

Fig. 3 The IEEE 9-bus test system 
Table 1 Parameters of the IEEE 9-bus system

\begin{tabular}{llllllllll}
\hline Circuit & $1-4$ & $2-7$ & $9-3$ & $5-4$ & $6-4$ & $7-5$ & $9-6$ & $7-8$ & $8-9$ \\
\hline Impedance (p.u.) & 0.0576 & 0.0625 & 0.0586 & 0.085 & 0.092 & 0.161 & 0.17 & 0.072 & 0.1008 \\
Resistance (p.u.) & 0 & 0 & 0 & 0.01 & 0.017 & 0.032 & 0.039 & 0.0085 & 0.0119 \\
MW limits (MW) & 150 & 200 & 100 & 100 & 120 & 150 & 100 & 100 & 100 \\
\hline
\end{tabular}

Table 2 Comparison of risk and cost for the IEEE 9-bus system

\begin{tabular}{llllll}
\hline Constraint & SCED & RBED & RB-SCED & \\
\cline { 3 - 6 } & & & $\operatorname{HSM}\left(K_{C}=1, K_{R}=0.5\right)$ & $\operatorname{ESM}\left(K_{C}=1.05, K_{R}=0.5\right)$ & $\operatorname{HEM}\left(K_{C}=1.20, K_{R}=0.5\right)$ \\
\hline Risk & 0.9 & 0.45 & 0.45 & 0.45 & 0.45 \\
Cost $(\$)$ & $11,377.8$ & $11,172.5$ & $11,679.3$ & $11,476.6$ & $11,172.5$ \\
\hline
\end{tabular}

Table 3 Comparison of generator outputs

\begin{tabular}{llll}
\hline Option & G1 $(\mathrm{WM})$ & G2 $(\mathrm{WM})$ & G3 $(\mathrm{WM})$ \\
\hline SCED & 136.46 & 161.60 & 21.40 \\
RBED & 146.76 & 142.88 & 29.82 \\
RB-SCED & & & \\
HSM & 136.46 & 154.83 & 28.17 \\
ESM & 143.06 & 148.14 & 28.26 \\
HEM & 146.76 & 142.88 & 29.82 \\
\hline
\end{tabular}

Table 4 Circuits with flow over $90 \%$ limit

\begin{tabular}{ll}
\hline Option & States of branch flow over $90 \%$ limit \\
\hline SCED & $100 \%$ limit, circuit $7-8$ at normal state \\
& $100 \%$ limit, circuit 6-4 at contingency 1 \\
RBED & $92.81 \%$ limit, circuit $7-8$ at normal state \\
& $112.5 \%$ limit, circuit 6-4 at contingency 1
\end{tabular}

RB-SCED

HSM $94.38 \%$ limit, circuit $7-8$ at normal state $100 \%$ limit, circuit 6-4 at contingency 1

ESM $\quad 93.75 \%$ limit, circuit $7-8$ at normal state $105 \%$ limit, circuit $6-4$ at contingency 1

HEM $92.81 \%$ limit, circuit $7-8$ at normal state

$112.5 \%$ limit, circuit 6-4 at contingency 1

The following are some discussions about the results:

1) Compared with SCED, RBED has less cost and fewer risk, but may cause high overloads for post-contingency states. For example, the flow on circuit 6-4 is $112.5 \%$ of its limit at contingency 1 .

2) HSM of RB-SCED is the most secure model but with the highest cost. Similar to SCED, it does not allow overload for post-contingency states, but its risk is only an half.
3) The cost of ESM model is close to SCED, but the risk is only $50 \%$. It decreases the power flow of circuit 7-8 at normal state from $100 \%$ limit to $93.75 \%$ limit, which permits relatively small post-contingency overloads (5\%) of circuit 6-4 at contingency 1 .

4) The results of the HEM model are the same to that of RBED. This happens because RBED is a special case to RB-SCED with $K_{C}$ being large enough. In this example, when $K_{C}$ is greater than 1.125 the dispatch result will not change.

\subsection{ISO New England system}

The proposed RB-SCED model was applied to the realtime power system in ISO New England, which consists of 12,300 buses, 13,500 circuits and 1,136 contingencies. The generator bidding data we used are from a winter day in 2009. Wind turbine units are not included since they do not have bidding curves. The number of bidding units is almost 400 .

The results presented here are tested by using MATLAB R2010a and CPLEX 12.1, on a $3.16 \mathrm{GHz}$ Intel Core $2 \mathrm{CPU}$ and 4 GB RAM PC. There are 802,150 decision variables and 4,002,196 constraints in the RB-SCED problem. The average time to solve this problem is 70 minutes. Table 5 compares the results between SCED and various cases of RB-SCED. By setting $K_{R}$ equals to 0.5 , we enforce the risk level of risk-based economic dispatch be half to SCED. The HSM case has higher operation cost nevertheless higher security level. The ESM and HEM cases have lower operating cost, but allows for a certain level of post-contingency overload. Since the probability of a occurring contingency is very low in real operation, they can bring significant economic benefits to the system. In addition, the post-contingency overload, whose level is controlled by 
Table 5 Results for the ISO New England system

\begin{tabular}{llllll}
\hline Constraint & SCED & RBED & RB-SCED & \\
\cline { 3 - 6 } & & & $\operatorname{HSM}\left(K_{C}=1, K_{R}=0.5\right)$ & $\operatorname{ESM}\left(K_{C}=1.05, K_{R}=0.5\right)$ & $\operatorname{HEM}\left(K_{C}=1.20, K_{R}=0.5\right)$ \\
\hline Risk & 18.2690 & 9.1345 & 9.1345 & 9.1345 & 9.1345 \\
Cost $(\$ / \mathrm{h})$ & $684,642.50$ & $605,407.32$ & $728,899.10$ & $610,611.54$ & $605,542.08$ \\
\hline
\end{tabular}

$K_{C}$, can be relieved by corrective actions during the dispatch. The application of RB-SCED in power systems is promising.

\section{Conclusion}

In this paper, the concept and the model of risk-based security-constrained economic dispatch (RB-SCED) are described. The risk is modeled as a product of contingency probability and overload severity. Compared with traditional SCED, the benefit of RB-SCED model lies in its ability to improve the economic performance of a power system while enhancing the system's overall security level, which has been demonstrated by testing in the IEEE 9-bus system and the ISO New England actual power system.

The following topics may be of interest for future research:

1) Testing the RB-SCED model on a real-world largescale power system.

2) Investigating how the objective function changes with parameters $K_{C}$ and $K_{R}$.

3) Developing a new locational marginal pricing (LMP) mechanism in accordance with the RB-SCED model.

Acknowledgements This work is jointly supported by National High Technology Research and Development Program of China (863 Program) (No. 2011AA05A105), and a key project from Zhejiang Electric Power Corporation.

Open Access This article is distributed under the terms of the Creative Commons Attribution License which permits any use, distribution, and reproduction in any medium, provided the original author(s) and the source are credited.

\section{References}

[1] Booth CJ (1993) The new IEEE standard dictionary of electrical and electronics terms, 5th edn. IEEE, New York, USA

[2] Balu N, Bertram T, Bose A et al (1992) On-line power system security analysis. Proc IEEE 80(2):262-279

[3] Weerasooriya S, El-Sharkawi MA, Damborg M et al (1992) Towards static-security assessment of a large-scale power system using neural networks. IEE Proc: Gener Transm Distr 139(1):64-70
[4] Laufenberg MJ, Pai MA (1997) A new approach to dynamic security assessment using trajectory sensitivities. In: Proceedings of 20th International Conference on Power Industry Computer Applications (PICA'97), Columbus, OH, USA, 11-16 May 1997, pp 272-277

[5] Billinton R, Gao Y, Karki R (2010) Application of a joint deterministic-probabilistic criterion to wind integrated bulk power system planning. IEEE Trans Power Syst 25(3):1384-1392

[6] Niebur D, Germond AJ (1992) Power system static security assessment using the Kohonen neural network classifier. IEEE Trans Power Syst 7(2):865-872

[7] Jia Z, Jeyasurya B (2000) Contingency ranking for on-line voltage stability assessment. IEEE Trans Power Syst 15(3):1093-1097

[8] La Commare KH, Eto JH (2004) Understanding the cost of power interruptions to US electricity consumers. LBNL-55718, Ernest Orlando Lawrence Berkeley National Laboratory, Berkeley, CA, USA

[9] Meliopoulos APS, Cokkinides GJ, Chao XY (1990) A new probabilistic power flow analysis method. IEEE Trans Power Syst 5(1):182-190

[10] Endrenyi J, Anders GJ, Leite da Silva AM (1998) Probabilistic evaluation of the effect of maintenance on reliability: an application to power systems. IEEE Trans Power Syst 13(2):576-583

[11] Allan R, Billinton R (2000) Probabilistic assessment of power systems. Proc IEEE 88(2):140-162

[12] Booth RR (1972) Power system simulation model based on probability analysis. IEEE Trans Power Appar Syst 91(1):62-69

[13] McCalley JD, Asgarpoor S, Bertling L et al (2004) Probabilistic security assessment for power system operations. In: Proceedings of the 2004 IEEE Power Engineering Society General Meeting, Vol 1, Dever, CO, USA, 6-10 Jun 2004, pp 212-220

[14] Kim H, Singh C (2005) Power system probabilistic security assessment using Bayes classifier. Electr Power Syst Res $74: 157-165$

[15] Ciapessoni E, Cirio D, Grillo S et al (2010) Operational risk assessment and control: a probabilistic approach. In: Proceedings of 1st IEEE PES Innovative Smart Grid Technologies Conference Europe (ISGT Europe'10), Milan, Italy, 11-13 Oct 2010, 8p

[16] Pereira MVF, Maceira MEP, Oliveira GC et al (1992) Combining analytical models and Monte-Carlo techniques in probabilistic power system analysis. IEEE Trans Power Syst 7(1):265-272

[17] Kirschen DS, Nedic DP, Allan RN (2004) A probabilistic indicator of system stress. IEEE Trans Power Syst 19(3):1650-1657

[18] Merrill HM, Wood AJ (1991) Risk and uncertainty in power system planning. Int J Electr Power Energ Syst 13(2):81-90

[19] Crousillat EO, Dorfner P, Alvarado P et al (1993) Conflicting objectives and risk in power system planning. IEEE Trans Power Syst 8(3):887-893

[20] McCalley JD, Fouad AA, Vittal V et al (1997) A risk-based security index for determining operating limits in stability-limited electric power systems. IEEE Trans Power Syst 12(3):1210-1219

[21] Kirschen DS, Jayaweera D (2007) Comparison of risk-based and deterministic security assessments. IET Gener Transm Distrib 1(4):527-533 
[22] He J, Cheng L, Kirschen DS et al (2010) Optimising the balance between security and economy on a probabilistic basis. IET Gener Transm Distrib 4(12):1275-1287

[23] McCalley JD, Vittal V, Abi-Samra N (1999) An overview of risk based security assessment. In: Proceedings of the IEEE Power Engineering Society Summer Meeting, Vol 1, Edmonton, Canada, 18-22 Jul 1999, pp 173-178

[24] Wan H, McCalley JD, Vittal V (2000) Risk based voltage security assessment. IEEE Trans Power Syst 15(4):1247-1254

[25] Ni M, McCalley JD, Vijay V et al (2003) On-line risk-based security assessment. IEEE Trans Power Syst 18(1):258-265

[26] Chen Q, McCalley JD (2005) Identifying high risk $N-k$ contingencies for online security assessment. IEEE Trans Power Syst 20(2):823-834

[27] Ni M, McCalley JD, Vijay V et al (2003) Online risk-based security assessment. IEEE Trans Power Syst 18(1):258-265

[28] Xiao F, McCalley JD (2007) Risk-based security and economy tradeoff analysis for real-time operation. IEEE Trans Power Syst 22(4):2287-2288

[29] Li Y, McCalley JD (2009) Risk-based optimal power flow and system operation state. In: Proceedings of the 2009 Power \& Energy Society General Meeting (PES'09), Calgary, Canada, 26-30 Jul 2009, 6p

[30] Xiao F, McCalley JD (2009) Power system risk assessment and control in a multi-objective framework. IEEE Trans Power Syst 24(1):78-87

[31] Dai R, Pham H, Wang Y et al (2012) Long term benefits of online risk-based DC optimal power flow. J Risk Reliab 226(1):65-74

[32] Wang Q, McCalley JD, Zheng T et al (2013) A computational strategy to solve preventive risk-based security constrained optimal power flow. IEEE Trans Power Syst 28(2):1666-1675

[33] Wang Q, McCalley JD. Risk and " $N-1$ " criteria coordination for real-time operations. Accept for publication by IEEE Trans Power Syst

[34] International Energy Agency (IEA). What can I do to save energy? http://www.iea.org/efficiency/whatcanido.asp

[35] Miranda V, Proenca LM (1998) Why risk analysis outperforms probabilistic choice as the effective decision support paradigm for power system planning. IEEE Trans Power Syst 13(2):643-648

[36] Linares P (2002) Multiple criteria decision making and risk analysis as risk management tools for power systems planning. IEEE Trans Power Syst 17(3):895-900

[37] Li W, Choudhury P, Gillespie D et al (2007) A risk evaluation based approach to replacement strategy of aged HVDC components and its application at BCTC. IEEE Trans Power Delivery 22(3):1834-1840

[38] Janjic A, Popovic D (2007) Selective maintenance schedule of distribution networks based on risk management approach. IEEE Trans Power Syst 22(2):597-604

[39] Strbac G, Moreno R, Pudjianto D et al (2011) Towards a riskbased network operation and design standards. In: Proceedings of the 2011 IEEE Power and Energy Society General Meeting, Detroit, MI, USA, 24-28 Jul 2011, 4p
[40] Zheng T, Litvinov E (2011) Operational risk management in the future grid operation. In: Proceedings of the 2011 IEEE Power and Energy Society General Meeting, Detroit, MI, USA, 24-28 Jul 2011, 3p

[41] Varaiya PR, Wu FF, Bialek JW (2011) Smart operation of smart grid: risk-limiting dispatch. Proc IEEE 99(1):40-57

[42] Xiao F, McCalley JD, Ou Y et al (2006) Contingency probability estimation using weather and geographical data for on-line security assessment. In: Proceedings of the International Conference on Probabilistic Methods Applied to Power Systems (PMAPS'06), Stockholm, Sweden, 11-15 Jun 2006, 7p

[43] McCalley JD, Xiao F, Jiang Y et al (2005) Computation of contingency probabilities for electric transmission decision problems. In: Proceedings of 13th International Conference on Intelligent Systems Application to Power Systems (ISAP'05), Arlington, TX, USA, 6-10 Nov 2005, pp 540-545

\section{Author Biographies}

Qin WANG (S'10) received his B.E., M.E. and Ph.D. degrees in Electrical Engineering from Huazhong University of Science and Technology (China), South China University of Technology (China), and Iowa State University (USA), in 2006, 2009 and 2012, respectively. His main research interests lie in economic dispatch and risk management of power systems.

Aimin YANG received his B.E. degree in electrical engineering from Zhejiang University, Hangzhou, China, and master degree in management from Fudan University, Shanghai, China, respectively. He is currently working for his Ph.D. degree in South China University of Technology. His main research interests lie in power system secure operation and electricity markets.

Fushuan WEN received the B.E. and M.E. degrees in electrical engineering from Tianjin University, Tianjin, China, in 1985 and 1988, respectively, and the Ph.D. degree from Zhejiang University, Hangzhou, China, in 1991. He joined the faculty of Zhejiang University in 1991, and has been a full professor and the director of the Institute of Power Economics and Information since 1997, and the director of Zhejiang University-Insigma Joint Research Center for Smart Grids since 2010. He had been a university distinguished professor, the deputy dean of the School of Electrical Engineering and the director of the Institute of Power Economics and Electricity Markets in South China University of Technology (SCUT), China, from 2005 to 2009 . His current research interests lie in power industry restructuring, power system alarm processing, fault diagnosis and restoration strategies, as well as smart grids.

Jihong LI received his B.E. and M.E. degrees in electrical engineering from Zhejiang University, Hangzhou, China. He has been working with Zhejiang Electric Power Corporation since 1985, and is current the deputy chief engineer of this company. His main research interests lie in power system dispatching and security. 